\title{
Lung Cancer Risk Associated with Regulated and Unregulated Chrysotile Asbestos Fibers
}

\author{
Ghassan B. Hamra, ${ }^{\mathrm{a}}$ David B. Richardson, ${ }^{\mathrm{b}}$ John Dement, ${ }^{\mathrm{c}}$ and Dana Loomis ${ }^{\mathrm{d}}$
}

\begin{abstract}
Background: Regulation of asbestos fibers in the workplace is partly determined by which fibers can be visually counted. However, a majority of fibers are too short and thin to count this way and are, consequently, not subject to regulation.

Methods: We estimate lung cancer risk associated with asbestos fibers of varying length and width. We apply an order-constrained prior both to leverage external information from toxicological studies of asbestos health effects. This prior assumes that risk from asbestos fibers increases with increasing length and decreases with increasing width.

Results: When we apply a shared mean for the effect of all asbestos fiber exposure groups, the rate ratios for each fiber group per unit exposure appear mostly equal. Rate ratio estimates for fibers of diameter $<0.25 \mu \mathrm{m}$ and length $<1.5$ and $1.5-5.0 \mu \mathrm{m}$ are the most precise. When applying an order-constrained prior, we find that estimates of lung cancer rate ratio per unit of exposure to unregulated fibers 20 40 and $>40 \mu \mathrm{m}$ in the thinnest fiber group are similar in magnitude to estimates of risk associated with long fibers in the regulated fraction of airborne asbestos fibers. Rate ratio estimates for longer fibers are larger than those for shorter fibers, but thicker and thinner fibers do not differ as the toxicologically derived prior had expected.

Conclusion: Credible intervals for fiber size-specific risk estimates overlap; thus, we cannot conclude that there are substantial differences in effect by fiber size. Nonetheless, our results suggest that some unregulated asbestos fibers may be associated with increased incidence of lung cancer.
\end{abstract}

(Epidemiology 2017;28: 275-280)

Submitted 22 February 2016; accepted 21 November 2016

From the a Department of Environmental and Occupational Health, Drexel University School of Public Health, Philadelphia, PA; ${ }^{b}$ Department of Epidemiology, University of North Carolina at Chapel Hill, Chapel Hill, $\mathrm{NC}$; 'Duke University Medical Center, Durham, NC; and ${ }^{\mathrm{d}}$ International Agency for Research on Cancer, Lyon, France.

D.B.R. was supported, in part, by Grant R03-OH-10706 from the National Institute for Occupational Safety and Health, Centers for Disease Control and Prevention.

The authors report no conflicts of interest.

The statistical software code is available from Dr. Hamra and as an eAppendix (http://links.lww.com/EDE/B147); however, authors must obtain permission to analyze the data.

SDC Supplemental digital content is available through direct URL citations in the HTML and PDF versions of this article (www.epidem.com).

Correspondence: Ghassan B. Hamra, Department of Environmental \& Occupational Health, Drexel University School of Public Health, 3215 Market St., Philadelphia, PA 19104. E-mail: ghassan.b.hamra@drexel.edu.

Copyright (C) 2016 Wolters Kluwer Health, Inc. All rights reserved. ISSN: $1044-3983 / 17 / 2802-0275$

DOI: 10.1097/EDE.0000000000000597
A sbestos has been classified by IARC as a human carcinogen since $1970 \mathrm{~s}^{1}$ and many countries have restricted or banned its production and use. Nevertheless, substantial production is ongoing in several middle-income industrial countries, including Brazil, India, China, and Russia; notably, it is widely used in Asia. ${ }^{2}$ Exposure also continues in countries that have restricted use; for example, approximately 1,000 tons of asbestos are imported in the United States each year and exposure to asbestos in industrial plants and in many buildings continues. ${ }^{3}$ Given that it is an established lung carcinogen $^{4,5}$ and occupational exposure persists, asbestos continues to pose important occupational hazards in the United States and worldwide.

Currently, permissible asbestos exposure levels in the workplace are expressed in terms of fibers of asbestos per milliliter of air (f/ml) averaged over an 8-hour period. ${ }^{6}$ Fibers are counted using phase contrast microscopy. Only asbestos particles greater than approximately $0.25 \mu \mathrm{m}$ (the resolution limit of phase contrast microscopy) and having an aspect ratio (i.e., a length:width ratio) of 3:1 or greater and a length greater than 5 $\mu \mathrm{m}$ are counted; the fibers that do not meet this criteria, because they are too thin and/or too short to be counted, are unregulated in workplace settings where asbestos fibers are subject to occupational safety standards. A persistent concern is that the specified dimensional criteria may not be sufficient for protecting the health of exposed workers because they are not based solely on health concerns. For example, the use of the $3: 1$ aspect ratio as the minimum to define a fiber was based on the ability of the microscopist to determine the elongate nature of a particle, ${ }^{7}$ and the practice has been carried through to this day. ${ }^{8}$ Airborne particles that are not counted as fibers by the standard are unregulated. These concerns would be largely academic if regulations to minimize the fiber concentrations always led to environments that had little or no airborne exposure to the unregulated fraction of fibers. However, prior work demonstrates that in most occupational and environmental settings nearly all of the airborne asbestos may be constituted by fibers with dimensions outside the range counted by phase contrast microscopy. ${ }^{9,10}$

Recent reviews suggest that the unregulated fibers may lead to occupational disease. ${ }^{11}$ Risk of asbestos-related disease may be greater for very thin fibers than for thicker fibers, and greater for long fibers than short fibers ${ }^{11}$; however, such conclusions likely depend on the health effect 
of interest and the epidemiologic evidence to support such conclusions is extremely sparse. In epidemiologic studies of occupational exposure to asbestos, estimation of the influence of dimensional attributes is complicated by the fact that workers are typically jointly exposed to particles of varying dimensional attributes, with correlation in concentrations of particles of differing dimensions. In prior studies, fiber sizespecific exposure estimates (based on combinations of fiber width and length) were examined one at a time and each was found to be a predictor of lung cancer and asbestosis. ${ }^{12,13}$ However, cumulative exposures were highly correlated across all fiber size categories, which complicates the interpretation of such results. Models to simultaneously estimate fiber size-specific effect estimates, by including terms for multiple length-diameter categories simultaneously, failed to converge in some analyses ${ }^{12}$ and yielded highly unstable estimates in other analyses. ${ }^{13}$ We previously modeled individual asbestos fibers in a single regression model using data from a cohort of North Carolina (United States) asbestos textile workers and Bayesian methods including shrinkage to a grand mean. The results suggested little difference across fiber size groups; however, we could not discount the possibility that there was not enough data to address this question and overcome high correlations among the asbestos groups. ${ }^{14}$

In the current article, we build on previous analyses of the North Carolina textile workers' cohort by pooling this group with a cohort of textile workers from South Carolina, both of which were exposed to chrysotile asbestos. We apply a hierarchical Bayesian model to jointly estimate the lung cancer risk associated with multiple asbestos fiber groups. Furthermore, we apply a model that implements an order constraint to integrate a prior belief that asbestos fibers of greater length and narrower diameter may be more harmful than shorter fibers.

\section{METHODS}

\section{Study Population}

Elliott et al. ${ }^{4}$ have previously studied the pooled cohort of North Carolina (NC) and South Carolina (SC) textile workers. We will briefly summarize the pooled cohort here, but provide reference to previous studies that detail characteristics of the pooled cohorts. ${ }^{15,16}$ The cohort includes 6,136 workers, 3,717 of whom were male. Workers were employed in a single facility in South Carolina (1,791 men, 1,263 women) or one of three facilities in North Carolina (1,926 men, 1,156 women). Of the three textile facilities in North Carolina, two produced asbestos beginning in the 1920s and the third since the 1940s. In South Carolina, production began in 1909 and ended in 1977. Exposure assessment methods have been thoroughly discussed and briefly summarized in numerous prior publications. ${ }^{10,14,17,18}$

Vital status was ascertained through 2001 in SC and through 2003 in NC. We observed 202 and 159 lung cancer deaths in SC and NC, respectively. In addition to a total of 361 observed lung cancer events, the combined cohorts provide a total of 218631 person-years at risk. Procedures for study of human subjects were approved by the Institutional Review Boards of the University of North Carolina, Chapel Hill.

Transmission electron microscopy was performed on a stratified random sample of 160 historical dust samples captured on membrane filters collected in surveys of the study plants in 1964-1971, as described previously. ${ }^{17,19}$ The transmission electron microscopy fiber-counting protocol was based on the International Standards Organization directtransfer method ${ }^{20}$ and procedures for combining the data by plant and operation and deriving size-specific exposure estimates followed the procedure described by Dement et al. ${ }^{10,17,19}$ Data from all of the study plants were modeled simultaneously by Poisson regression to estimate adjustment factors as proposed by Quinn et al. ${ }^{21}$ for each length-diameter category. ${ }^{10,17,19}$ These were applied to a matrix of plant-, department- and time-specific fiber concentrations estimated by the standard phase contrast microscopy (PCM) method to generate fiber size-specific estimates of exposure. ${ }^{10}$ TEM estimates exposure to asbestos fibers in four categories of fiber width $(<0.25,0.25-1,1-3$, and $>3 \mu \mathrm{m})$ and six categories of fiber length $(<1.5,1.5-5,5-10,10-20,20-40,40+\mu \mathrm{m})$. Estimated exposures to fibers of different dimensions were linked to workers' occupational histories for assignment of individual cumulative exposure measured in fiber-years $/ \mathrm{ml}(\mathrm{f}-\mathrm{y} / \mathrm{ml})$.

There were no fibers counted in three of the 24 possible categories of diameter, length $1.0-3.0,<1.5 \mu \mathrm{m} ; 3.0-5.0,<1.5$ $\mu \mathrm{m}$; and 3.0-5.0, 1.5-5.0 $\mu \mathrm{m}$. Therefore, in the current analysis, we consider estimates of cumulative asbestos exposure in 21 length/diameter groups. Furthermore, we note that the vast majority of asbestos used in these plants was raw chrysotile. Small amounts of crocidolite yarn were used in the SC plant (1950-1975) and some amosite was used in one of the North Carolina plants (1963-1976). Thus, results are with regard to chrysotile asbestos. ${ }^{4}$

\section{Statistical Analyses}

We conduct a nested case-control study using incidence density sampling with 10 controls matched to each case by age at the time of lung cancer event. We adjust models for continuous age, sex (male, female), race (white, non-white), and year of observation in ordinal categories from 1950 to 1959 (reference), 1960 to 1969,1970 to 1979,1980 to 1989,1990 to 1999 , and $2000+$. The model follows the form

$\operatorname{logit}(P(D=1))=\alpha+\sum_{i=1}^{I} \beta_{i} x_{i}+\varphi_{1} * \operatorname{age}+\varphi_{2} *$ race $+\varphi_{3} * \operatorname{sex}+\varphi_{4} *$ year

in which $\beta_{i}$ represents the change in the logit(risk) of lung cancer per unit increase in exposure to cumulative asbestos in 1 of 21 length/diameter groups. Cumulative exposures are lagged 10 years. Elliott et al. ${ }^{4}$ demonstrated that changes to the assigned lag, ranging 
from 0 to 20 years, did not result in meaningful changes to the risk estimates (eAppendix; http://links.lww.com/EDE/B147).

Toxicologic evidence from animal models provides fairly strong support for the notion that the risk of asbestosrelated disease is greater for longer, thinner fibers compared with shorter, thicker fibers. ${ }^{22-24}$ However, critical reviews of the literature concentrating on lung tissue burden analyses suggest that it is difficult to exclude fibers of any particular dimension from a role in causing disease. ${ }^{11}$ Furthermore, asbestos fibers of all shapes and size have been classified as group 1 carcinogens by the International Agency for Research on Cancer. There are differing views concerning the role of short $(<5 \mu \mathrm{m})$ fibers. ${ }^{25}$ While studies in animals are subject to uncertainties with respect to how their findings apply to humans, the evidence from such studies serve as the basis for a somewhat informative prior regarding the effects of fiber size. In the current study, we apply a prior to the data in the form of a simple ordered constraint on the parameters $\beta_{i}$. Within a category of fiber width, the ordered constraint imposes the assumption that the parameter describing the effect of fiber length category $n+1$ is equal to or greater than the parameter for fiber length $n$. Furthermore, we apply a second constraint that assumes a greater effect of fibers in diameter category $m-1$ relative to category $m$; in other words, thinner fibers are believed to be more carcinogenic than thicker fibers. Finally, we impose a constraint that no asbestos fiber can protect an individual from developing lung cancer (i.e., negative values for $\boldsymbol{\beta}_{i}$ are implausible). These constraints imply monotonicity of effect (biologic effectiveness may not reverse across the range of support in the observed data) but are flexible with regards to shape of the function and magnitude of effects regarding relative effectiveness of fiber size. We compare results from this model to one in which no order constraints are imposed, but in which we assume that the rate ratio of lung cancer due to exposure to asbestos fibers of a specific size may share a common mean (i.e., that the effects are partially exchangeable), as in previous analyses of the North Carolina asbestos cohort. ${ }^{14}$ In brief, this model assumes the effect for any individual fiber size group, $\boldsymbol{\beta}_{i}$, arises from the same distribution as other fibers so that $\boldsymbol{\beta}_{i} \sim N\left(\mu, \tau^{2}\right)$. The prior on $\tau^{2}$ is largely noninformative so that the data determine the degree to which effect estimates are shrunk toward the group mean.

Models are run for 100,000 iterations with a burn-in of 10,000 iterations and thinning $=2$. We utilize a Gelman-Rubin diagnostic to assess convergence over two chains, after which the estimates from the two chains are pooled. ${ }^{26}$ Bayesian models are fit with the Just Another Gibbs Sampler (JAGS) ${ }^{27}$ package. We note that for order-constraint model, we rerun analyses including a more and less informative prior on the variance of the coefficients, and results appear nearly identical. Furthermore, we run models in which we impose only a constraint on length but not diameter. Results are similar to the order-constrained model we apply here and, thus, results are not presented.

\section{RESULTS}

Table 1 provides descriptive characteristics of the North Carolina (NC) and South Carolina (SC) cohorts, as well as the pooled cohort. A higher percentage of workers in the SC cohort were white, and were of slightly younger age at first employment. Also, workers in SC were employed for a longer time period and contributed more total person-years at risk. Notably, exposures were much higher in the NC plants compared with the SC plant, with an average cumulative fiber-ml/ year exposure of 80.4 compared with 28.2.

Table 2 summarizes estimates from the models described above and presents prevalence of each fiber size group in the airborne samples analyzed by transmission electron microscopy. The smallest fibers (i.e., $<0.25 \mu \mathrm{m}$ in diameter and $<1.5$ $\mu \mathrm{m}$ in length) represent $54 \%$ of the total fiber count. Fibers in the largest fiber size group by diameter $(>3.0 \mu \mathrm{m})$ represent the smallest fraction of total fibers; in sum, these fibers are less than $0.4 \%$ of the total fibers counted.

When we apply a shared mean to all asbestos fiber groups, the rate ratio associated with all fiber size groups appear equal in magnitude. We note that the rate ratio for the shared mean estimated by the data was 1.02 . In the case of the model subject to the order constraints, the risk of lung cancer increases with increasing length, but does not follow the prior assumption that risk will increase with decreasing diameter. In both models, the most precise estimates are obtained for asbestos fibers with diameter $<0.25 \mu \mathrm{m}$ and length $<1.5 \mu \mathrm{m}$ or between 1.5

TABLE 1. Characteristics of a Combined Cohort of 6,136 Asbestos Textile Workers from Plants Located in North Carolina (NC) and South Carolina (SC) (1940-2003)

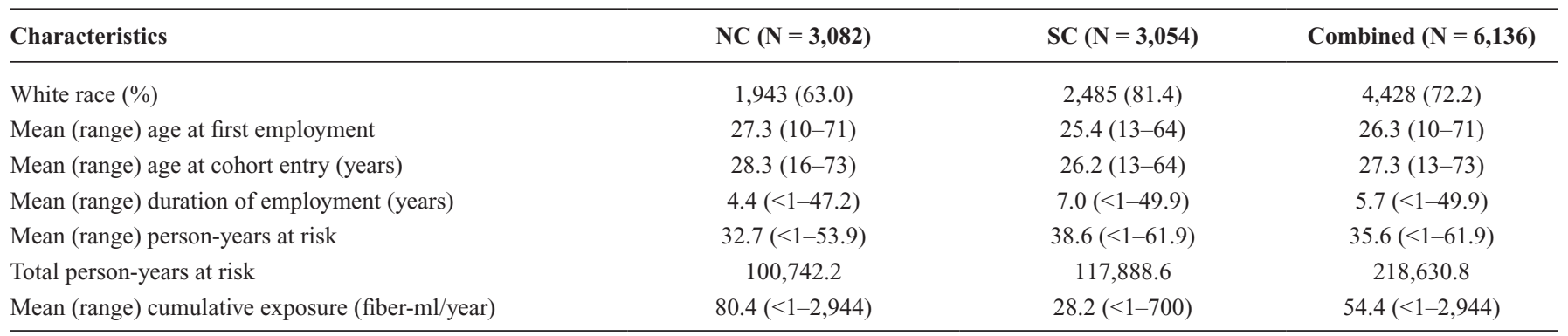


TABLE 2. Coefficients for Change in the RR of Lung Cancer Associated with a $100 \mathrm{f}-\mathrm{yr} / \mathrm{ml}$ Change in Exposure to Chrysotile Asbestos, by Diameter and Length Groups, in a Cohort of North and South Carolina Asbestos Textile Workers

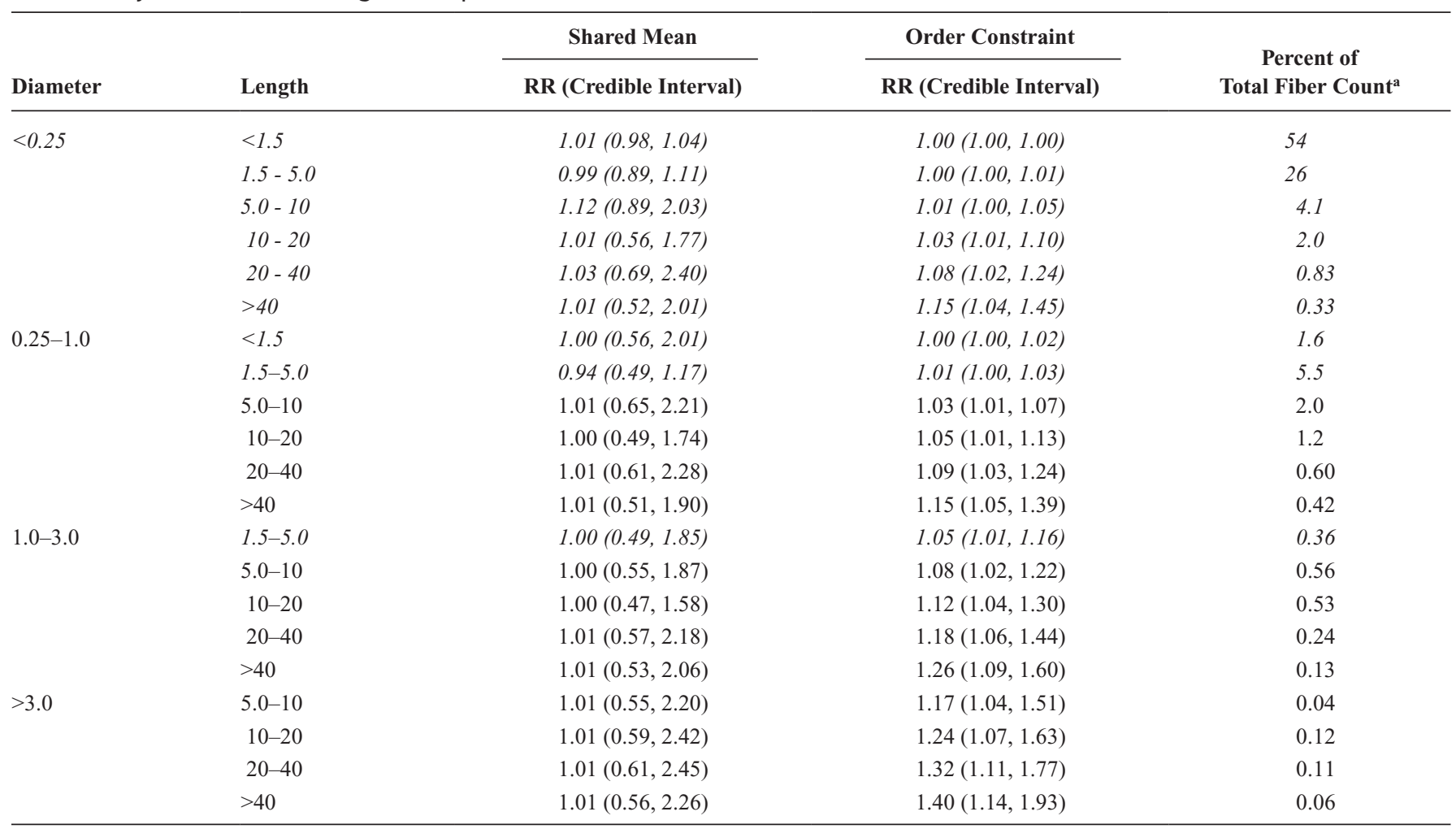

Italicized values indicate unregulated diameter/length groups for asbestos fibers. The regression model is adjusted for age (continuous), sex, race, and year of employment. ${ }^{\mathrm{a}}$ Mean proportion of fibers for all plants and operations combined. ${ }^{10}$

$\mathrm{RR}$ indicates rate ratio.

and $5.0 \mu \mathrm{m}$, and are at or near the null. In fact, estimates for the thinnest $(<0.25 \mu \mathrm{m})$ fiber size group are the most precise in both models. Risk estimates become less precise with increasing width and length. The asbestos fiber groups with the largest risk estimates are also the least precise in all diameter groups, especially those for fibers of width $>3.0 \mu \mathrm{m}$.

The Figure displays fiber size-specific estimates reported in Table 2 for the model in which we assume that rate ratio estimates may share a common mean effect (left column) and for the model in which we assume the order constraints based on toxicology (right column). Again, it is clear that the association between asbestos fiber exposure and lung cancer mortality increases with increasing fiber length, but does not increase as diameter decreases. The shared mean model suggests little difference in risk imposed by fiber size category whereas the model with ordered constraints found that the constraint on length had a greater impact than the constraint on width, suggesting that the data are more supportive of the former.

\section{DISCUSSION}

Current regulation of asbestos fibers in the workplace is informed by scientific evidence and limitations in the ability to measure shorter, thinner fibers with standard methods. This has led to a policy that does not directly consider the health effects of shorter, thinner fibers. However, our analyses integrating evidence from toxicology suggest that unregulated fibers have a meaningful effect on lung cancer mortality. Our analysis supports and strengthens prior analyses that considered only the cohort of North Carolina textile workers. ${ }^{28}$ Importantly, using a Bayesian approach, we were able to jointly estimate effects for all fiber size groups in a single model while also incorporating evidence from toxicology regarding relative effects of asbestos fibers by fiber length and diameter. The order constraint imposes a strong assumption that lung cancer risks associated with asbestos fibers increase with increasing length and with decreasing width; this prior relies on the belief that animal models can inform observational studies in humans. We emphasize that the constraint of increasing risk with increasing length seemed to outweigh the constraint of increasing risk with decreasing width. We believe this is because the data provide more support for an effect of length versus width. Credible intervals for fiber size-specific rate ratio estimates overlap; thus, we cannot firmly conclude that there are substantial differences in effect by fiber size. Nonetheless, our results suggest that some unregulated asbestos fibers are likely carcinogenic.

Several issues should be considered with regard to interpretation of the estimated risk posed by fibers of varying lengths based on our model results. First and perhaps most important, 

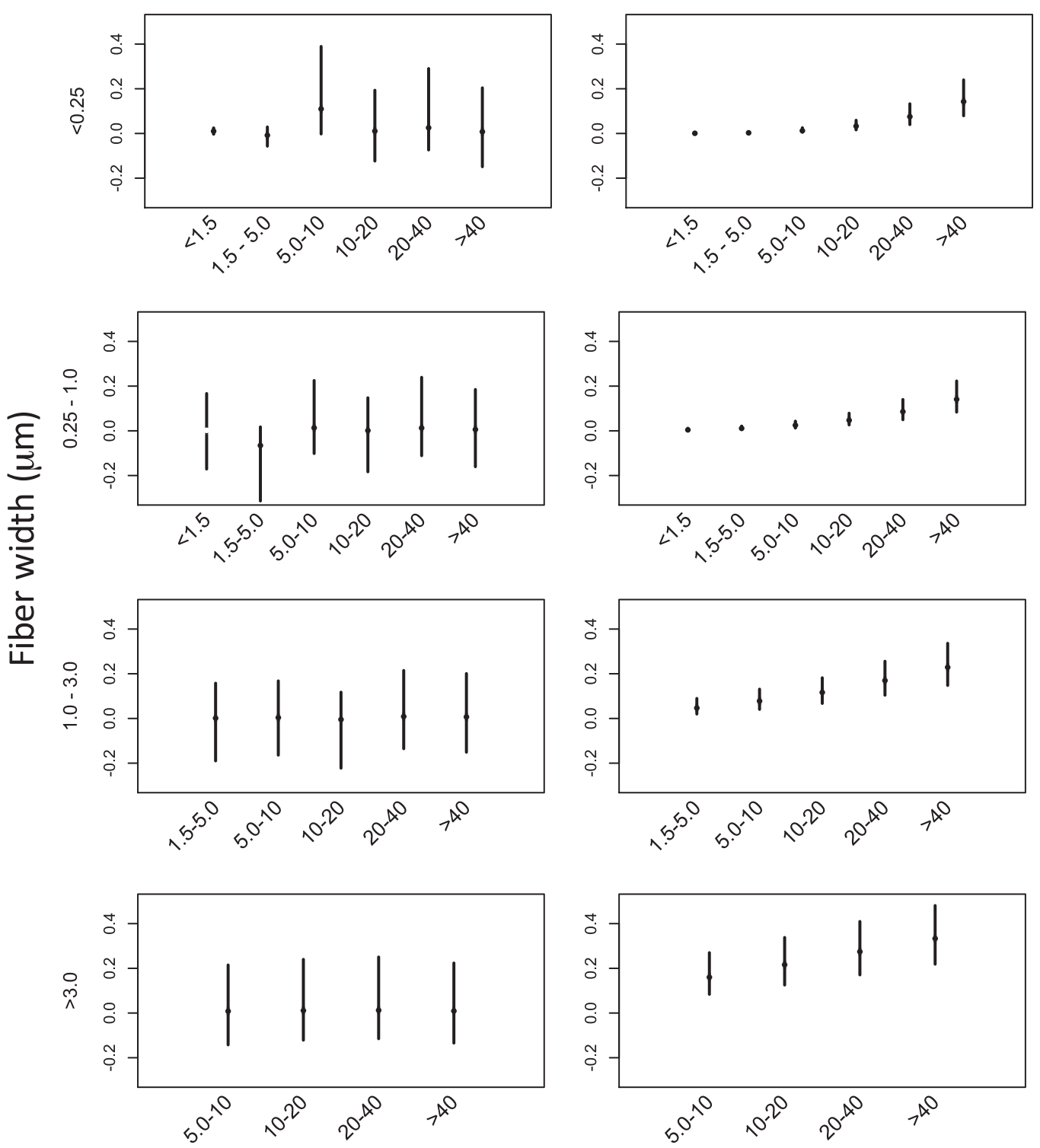

Fiber length $(\mu \mathrm{m})$

FIGURE. Medians and estimated SDs of coefficients $\left(\beta_{i}\right)$ for chrysotile fiber size groups subject to a shared mean (left) or order constraints (right). Lines represent 16th-84th percentile values of the distribution.

the overall risk of lung cancer among these workers is the result of all inhaled fibers acting collectively. Both our shared mean and order-constrained models attempted to disaggregate the effects by size and the rate ratios presented by fiber size are based on increased rate per $100 \mathrm{f}$-year/ml (Table 2). As shown in Table 2, unregulated short and thin fibers (i.e., $<0.25 \mu \mathrm{m}$ in diameter and $<5.0 \mu \mathrm{m}$ in length) account for approximately $79 \%$ of all fibers to which workers were exposed. Rate ratio estimates for asbestos fibers $<5 \mu \mathrm{m}$ were generally of much lower magnitude compared with longer fibers. However, the decrease in the magnitude of the rate ratio per unit exposure for shorter fibers should be considered with respect to their sheer numbers in the airborne dust. Second, fiber respirability is largely governed by fiber diameter. ${ }^{29,30}$ Smaller diameter fibers are most respirable and fibers $>3.0 \mu \mathrm{m}$ in diameter have a lower probability of deposition beyond the nasopharyngeal and tracheobronchial regions with subsequent mucociliary clearance. While our models found higher rate ratio estimates for fibers $>3.0 \mu \mathrm{m}$ in diameter in the size constrained model, their presence in the airborne dust is relatively rare and those that were present would have a lower probability of deposition at critical sites. The same comment applies to several other fiber size categories that represent a small proportion of the fiber exposures experienced by these workers. Finally, neither of our models nor previous analyses of specific fiber size ranges ${ }^{18,28}$ exclude a contribution of short and thin fibers to the risk of lung cancer. 
Consideration of health effects of asbestos fibers by size is important because regulating only a subset of asbestos fibers may be inadequate for protecting human health. Importantly, asbestos fibers of varying dimensions are created in the processing of raw materials and large portions of these fibers are in the smallest size categories that are currently unregulated. Unfortunately, techniques that prevent worker exposures to regulated fibers do not necessarily limit or eliminate worker exposure to unregulated fibers. Personal protective equipment may not be capable of preventing inhalation and exposures to these smallest fibers, which are in the range of nanoparticles. This study suggests that protection standards should be adapted to minimize or eliminate exposures to asbestos fibers of any size.

Our analyses leverage the information available in the data and external to the data by integrating toxicology evidence with Bayesian methods. These methods are an essential tool in considering the joint effects of highly correlated exposures $^{31,32}$; however, like any other method, it is not always possible to overcome limitations in the data. The asbestos fiber size groups considered here are highly correlated. In some instances, fiber size groups are nearly perfectly correlated, which may explain the similarity in results when we impose partial exchangeability of effects in our analyses presented in Table 2 across asbestos fiber groups. We believe this limits the ability to consider the health effects of any one asbestos fiber size group outside of a controlled, experimental setting.

Asbestos is classified as a known carcinogen by the International Agency for Research on Cancer, regardless of its form. ${ }^{1}$ Our results integrating toxicologic evidence suggest that longer, thicker fibers are as carcinogenic as longer, thinner fibers. Our results are consistent with the conclusions of the International Agency for Research on Cancer that asbestos is carcinogenic. ${ }^{33}$

\section{REFERENCES}

1. (IARC) International Agency for Research on Cancer. A Review of Human Carcinogens: Arsenic, Metals, Fibres, and Dusts. Lyon, France: International Agency for Research on Cancer; 2012.

2. Virta RL, Flanagan DM. 2014 Minerals Yearbook. In: USGS, ed. Reston, VA: United States Geological Survey; 2015.

3. Camus M, Siemiatycki J, Meek B. Nonoccupational exposure to chrysotile asbestos and the risk of lung cancer. N Engl J Med. 1998;338:1565-1571.

4. Elliott L, Loomis D, Dement J, Hein MJ, Richardson D, Stayner L. Lung cancer mortality in North Carolina and South Carolina chrysotile asbestos textile workers. Occup Environ Med. 2012;69:385-390.

5. National Toxicology Program (US). Report on carcinogens background document for Silica Crystalline (Respirable Size). Research Triangle Park, NC: National Toxicology Program.

6. (ATSDR) Agency for Toxic Substances and Disease Registry. Toxicological Profile for Asbestos. Atlanta, GA: US Department of Health and Human Services, Public Health Service; 2001.

7. Holmes S. Developments in dust sampling and counting techniques in the asbestos industry. Ann NY Acad Sci. 1965;132:288-297.

8. Chu H, Gange SJ, Yamashita TE, et al. Individual variation in CD4 cell count trajectory among human immunodeficiency virus-infected men and women on long-term highly active antiretroviral therapy: an application using a Bayesian random change-point model. Am J Epidemiol. 2005;162:787-797.
9. Dement JM. Workshop on fiber toxicology research needs. Environ Health Perspect. 1990;88:261-268.

10. Dement JM, Loomis D, Richardson D, Wolf SH, Kuempel ED. Estimates of historical exposures by phase contrast and transmission electron microscopy for pooled exposure--response analyses of North Carolina and South Carolina, USA asbestos textile cohorts. Occup Environ Med. 2011;68:593-598.

11. Dodson RF, Atkinson MA, Levin JL. Asbestos fiber length as related to potential pathogenicity: a critical review. Am J Ind Med. 2003;44:291-297.

12. Lunn M, McNeil D. Applying Cox regression to competing risks. Biometrics. 1995;51:524-532.

13. Greenland S. Quantifying biases in causal models: classical confounding vs collider-stratification bias. Epidemiology. 2003;14:300-306.

14. Hamra GB, Loomis D, Dement J. Examining the association of lung cancer and highly correlated fibre size-specific asbestos exposures with a hierarchical Bayesian model. Occup Environ Med. 2014;71:353-357.

15. Loomis D, Dement J, Richardson D, Wolf S. Asbestos fibre dimensions and lung cancer mortality among workers exposed to chrysotile. Occup Environ Med. 2010;67:580-584.

16. Loomis D, Dement JM, Wolf SH, Richardson DB. Lung cancer mortality and fibre exposures among North Carolina asbestos textile workers. Occup Environ Med. 2009;66:535-542.

17. Dement JM, Myers D, Loomis D, Richardson D, Wolf S. Estimates of historical exposures by phase contrast and transmission electron microscopy in North Carolina USA asbestos textile plants. Occup Environ Med. 2009;66:574-583.

18. Stayner L, Kuempel E, Gilbert S, Hein M, Dement J. An epidemiological study of the role of chrysotile asbestos fibre dimensions in determining respiratory disease risk in exposed workers. Occup Environ Med. 2008;65:613-619.

19. Dement JM, Kuempel ED, Zumwalde RD, Smith RJ, Stayner LT, Loomis D. Development of a fibre size-specific job-exposure matrix for airborne asbestos fibres. Occup Environ Med. 2008;65:605-612.

20. International Standards Organisation. Ambient air--determination of asbestos fibres--direct transfer electron microscopy method. Geneva, 1995. ISO 10312.

21. Quinn MM, Smith TJ, Eisen EA, Wegman DH, Ellenbecker MJ. Implications of different fiber measures for epidemiologic studies of man-made vitreous fibers. Am J Ind Med. 2000;38:132-139.

22. Lippmann M. Asbestos exposure indices. Environ Res. 1988;46:86-106.

23. Berman DW, Crump KS. A meta-analysis of asbestos-related cancer risk that addresses fiber size and mineral type. Crit Rev Toxicol. 2008;38(suppl 1):49-73.

24. Berman DW, Crump KS, Chatfield EJ, Davis JM, Jones AD. The sizes, shapes, and mineralogy of asbestos structures that induce lung tumors or mesothelioma in AF/HAN rats following inhalation. Risk Anal. 1995; 15:181-195.

25. Gwinn MR, DeVoney D, Jarabek AM, et al. Meeting report: mode(s) of action of asbestos and related mineral fibers. Environ Health Perspect. 2011;119:1806-1810.

26. Hamra G, MacLehose R, Richardson D. Markov chain Monte Carlo: an introduction for epidemiologists. Int J Epidemiol. 2013;42:627-634.

27. JAGS: A program for analysis of Bayesian graphical models using Gibbs sampling [computer program]. 2003.

28. Loomis D, Dement JM, Elliott L, Richardson D, Kuempel ED, Stayner L. Increased lung cancer mortality among chrysotile asbestos textile workers is more strongly associated with exposure to long thin fibres. Occup Environ Med. 2012;69:564-568.

29. Lippmann M. Effects of fiber characteristics on lung deposition, retention, and disease. Environ Health Perspect. 1990;88:311-317.

30. Harris RL Jr, Timbrell V. The influence of fibre shape in lung depositionmathematical estimates. Inhaled Part. 1975;4(Pt 1):75-89.

31. Maclehose RF, Dunson DB. Bayesian semiparametric multiple shrinkage. Biometrics. 2010;66:455-462.

32. MacLehose RF, Dunson DB, Herring AH, Hoppin JA. Bayesian methods for highly correlated exposure data. Epidemiology. 2007;18:199-207.

33. OSHA. Final Rules, Occupational Exposure to Asbestos, Standard Number: 1910.1001; 1915:1001; 1926.58. 1994. 